\title{
Advances in the Combination Therapy of Targeted Anti-Cancer Drugs
}

\author{
Jie Yang*, Yan Wu, Zhen Wu, Yong-Tao Cui, Xiao-Qi Peng \\ Patent Examination Cooperation Center of the Patent Office, SIPO, Beijing, China
}

\section{Email address:}

yangjie_3@sipo.gov.cn (Jie Yang),wuyan_1@sipo.gov.cn (Yan Wu), wuzheng@sipo.gov.cn (Zhen Wu), cuiyongtao@sipo.gov.cn (Yong-Tao Cui), pengxiaoqi@sipo.gov.cn (Xiao-Qi Peng)

${ }^{*}$ Corresponding author

\section{To cite this article:}

Jie Yang, Yan Wu, Zheng Wu, Yong-Tao Cui, Xiao-Qi Peng. Advances in the Combination Therapy of Targeted Anti-Cancer Drugs. Science Discovery. Vol. 4, No. 2, 2016, pp. 94-98. doi: 10.11648/j.sd.20160402.17

Received: April 27, 2016; Accepted: May 16, 2016; Published: May 18, 2016

\begin{abstract}
The combination therapy of targeted anti-cancer drugs is important in the research of anti-cancer drugs. This articleintroduced the status of combination therapy of the same targeted and different targeted anti-cancer drugs, and analyzed the challenges and difficulties. In order to provide reference basis for development of combination therapy technique of targeted anti-cancer drugs in China.
\end{abstract}

Keywords: Cancer, Drug, Target, Combination Therapy

\section{癌症靶向药物联合治疗的技术现状及发展趋势}

杨杰*，吴燕，吴峥，崔永涛，彭晓琦

国家知识产权局专利局专利审查协作北京中心，北京，中国

\section{邮箱}

yangjie_3@sipo.gov.cn（杨杰）, wuyan_1@sipo.gov.cn（吴燕）, wuzheng@sipo.gov.cn（吴峥）,

cuiyongtao@sipo.gov.cn（崔永涛）, pengxiaoqi@sipo. gov.cn（彭晓琦）

摘要: 癌症靶向药物联合治疗是抗癌药物研究的重要方向。本文从联合用药抑制同一特异性靶点和联合使用不同靶点 的药物两方面对癌症联合靶向治疗技术现状进行介绍，并对联合靶向治疗技术发展面临的挑战和困难作了分析，以期 为中国癌症靶向药物联合治疗技术的发展提供参考依据。

关键词：癌症，药物，靶向，联合治疗

\section{1. 引言}

肿瘤作为一种恶性疾病严重威胁着人类健康, 发病率 居高不下。据WHO预测，至2030年全球将有超过1700万人 死于癌症 ${ }^{[1]}$ 。在中国, 癌症已成为第二大死亡原因和城市 的首位死因。在癌症的治疗中, 分子靶向治疗是以肿瘤细 胞过度表达的某些标志性分子为靶点, 选择针对性的阻断
剂, 以有效干预受该标志性分子调控并与肿瘤发生密切相 关的信号传导通路, 从而达到抑制肿瘤生长、进展及转移 的效果。靶向药物的应用不但增强了治疗的特异性和选择 性, 且避免了一般化疗药物的无选择性毒副作用和耐药性。 由于肿瘤的发生发展不是单一因素的结果, 而是多因素多 基因多靶点多环节的调控过程, 企图阻断一个受体而阻断 任何信息传导的做法显然不可能产生持久的生长抑制, 因 
此导致单一靶分子的治疗往往需要较大剂量, 仍有一定毒 性, 疗效有限。可见, 肿瘤形成和转移涉及的复杂分子网 络系统异常说明肿瘤靶向治疗中联合用药的必要性和重 要性。

至今已有的癌症联合靶向治疗大致归纳为以下两大 类: 联合用药最大抑制同一特异性靶点, 如单克隆抗体和 小分子酪氨酸激酶抑制剂共同作用于表皮生长因子受体 (EGFR); 联合使用不同靶点的药物以达到最大的抗肿瘤作 用, 如BEGFR抑制剂和血管上皮生长因子受体抑制剂联合 使用, EGFR、RAS和RAF抑制剂联合使用等。以下分别从这 两类癌症联合靶向治疗技术进行介绍:

\section{2. 联合用药抑制同一特异性靶点}

经过几十年的研究, 目前人类对肿瘤的细胞生物学和 遗传学方面的认识有了飞速发展, 一系列重大发现包括癌 基因、抑癌基因、细胞凋亡、肿瘤血管形成等使癌症研究 由细胞生物学水平转变到分子生物学水平, 一系列新的概 念包括信号传导、细胞周期、DNA修复等已经得到验证, 以此为基础, 大量以肿瘤的分子遗传学改变及其在肿瘤细 胞水平的表达为靶点的抗肿瘤药物不断被研发, 主要包括 细胞信号传导分子抑制剂、血管新生抑制剂、靶向端粒酶 的抑制剂和针对肿瘤耐药的逆转剂, 其中分子靶点涉及蛋 白激酶、肿瘤血管生成因子、DNA拓扑异构酶、其它蛋白 酶例如泛素蛋白酶等。为达到最大限度地抑制单个关键靶 点的目的, 针对同一靶点机理不同的靶向药物已经被联合 使用 ${ }^{[2]}$ 。

以EFGR为例, 靶向EGFR主要有两类药物 ${ }^{[3]}$, 一种是针 对EGFR胞外部分的单克隆抗体, 代表药物西妥昔单抗是第 一种被美国FDA批准用于已发生转移的晚期直肠癌患者治 疗的单克隆抗体, 其作用机制为: 与EGF、TGF等配体竞争 EGFR分子上的特殊结合位点, 阻断配体对受体的激动作用, 并通过EGFR的内吞、失活, 下调其在胞膜的表达水平, 还可 激活抗体依赖的细胞毒作用 (ADCC), 产生进一步的细胞杀 伤效应; 另一种是针对EGFR胞内部分的小分子酪氨酸激酶 抑制剂, 代表药物为吉非替尼和厄洛替尼, 其作用机制为 与ATP竞争GFR胞内部分酪氨酸激酶结构域上的ATP结合位 点, 阻断由EGFR活化引起的一系列胞内级联酶促反应, 阻 止活化信号转导至核内。由于作用位点的差异, 两类药物 的联合使用可以克服各自单用的局限性, 具有积极的意义。

以下是包括靶向EFGR在内的一些具体研究的实例:

1. 联合使用作为EGFR中和抗体的西妥昔单抗和作为 EGFR酪氨酸激酶抑制剂的厄洛替尼, 对于EGFR突变阴性的 NSCLC（非小细胞肺癌）患者无效, 但对于T790M突变阳性 的继发性耐药的NSCLC患者有效 ${ }^{[4]}$; 并且相同两种药物联 合应用于人肝癌细胞系的体外作用时, 具有明显的协同效 应, 从胞外和胞内两个层次上阻断了EGFR的活化, 进一步 下调了其下游信号转导通路中活化的关键酶蛋白的表达。 经西妥昔单抗和厄洛替尼单独或联合处理后的HepG2和 Be1-7402细胞, EGFR、ERK1/2、AKT的表达较对照组和EGF 处理组相比无显著性差异, 但是其活化形式: $p-E G F R 、$ $\mathrm{p}-\mathrm{ERK} 1 / 2 、 \mathrm{p}-\mathrm{AKT}$ 的表达均有不同程度的下调, 两药联用 $48 \mathrm{~h}$ 后, $\mathrm{p}-\mathrm{ERK} 1 / 2$ 和p-AKT的表达几乎完全受到抑制 ${ }^{[5]}$ 。
2. 联合使用作为VEGF中和抗体的贝伐珠单抗和作为 VEGF受体抑制剂的索拉非尼/舒尼替尼, 可最大限度地抑 制VEGF-VEGFR信号。并且, 由于受体抑制剂可诱导VEGF, 加用贝伐珠单抗会阻止潜在的负反馈回路, 可增强靶效应。 VEGF抑制剂的不良反应在联合使用中进一步突出, 需要进 一步减少剂量; 其中使用贝伐珠单抗与舒尼替尼组合治疗 RCC患者副作用较多。考虑单药的活性一般, 联合用药阻 断VEGF通路对卵巢癌和RCC具有更明显抗肿瘤活性 ${ }^{[6]}$ 。

3. 联合使用作为HER2 中和抗体的曲妥珠单抗和作为 HER2/EGFR酪氨酸激酶抑制剂的拉帕替尼, 对于先前接受 以曲妥珠单抗为基础方案治疗的、疾病仍有进展的ErB2 阳性、曲妥珠单抗抵抗的转移性乳腺癌患者, 能显著改善 无进展生存 (PFS) 和临床获益率 (CBR) ${ }^{[7]}$ 。

从现有的研究发现, 针对相同靶点的药物联合比独立 通路的靶点药物联合更容易有过多的不良反应, 此外, 相 对作用于相同靶点的小分子酪氨酸激酶抑制剂而言, 单克 隆抗体似乎更容易联合, 因为其药代动力学相互作用的趋 势或靶外效应的可能性低。在疗效方面, 尽管所有的试验 设计基于明确的理由, 但结果往往是不可预测的, 已证明 有些联合方案有疗效, 但也有失败的案例。

\section{3. 联合使用不同靶点的药物}

由于健康细胞同样依赖于癌症中激活的信号传导途 径得以存活, 如果在癌症治疗过程中抑制了这些途径会导 致对健康细胞产生过多的损害, 而癌细胞则可通过依赖过 度活跃的信号传导得以存活，从而产生有害的副作用。而 联合使用不同靶点的药物可通过将药物作用于细胞信号 网络的多个节点, 则可能更精确地发挥药物靶向的精确性, 并克服抗药性问题, 从而达到良好的治疗效果。

\subsection{AKT抑制剂和p $70 \mathrm{~S} 6 \mathrm{~K} 1$ 抑制剂的联用}

铂族金属抗癌药物如顺铂属于细胞周期非特异性药 物, 顺铂类抗癌药物可与DNA上的鸟嘌呤、腺嘌呤、胞嘧 啶形成交叉联结破坏DNA的结构和功能, 从而通过与DNA 分子形成链内或链间交叉联接或阻止RNA分子再复制等途 径发挥抗肿瘤药理作用。江秉华等人以作为铂类药物的代 表作为研究对象, 发现AKT扩增和过表达通过其下游作用 的靶分子p70S6K1调节肺癌、前列腺癌和卵巢癌细胞产生 耐药, 从而基于AKT/p70S6K1信号通路的激活是肿瘤细胞 对铂类药物产生获得性耐药的重要分子机制的认识开发 了一种抗肿瘤组合物, 该药物含有治疗有效量的铂类药物 以及AKT抑制剂和/或p70S6K1抑制剂。应用AKT和p70S6K1 的抑制剂与铂类药物合用, 不但可能减少铂类药物的用量, 而且能够延缓耐药发生和逆转肿瘤细胞获得耐药性 ${ }^{[8]}$ 。

\section{2. PI3K途径垂直通路的联合治疗}

PI3K（磷脂酰肌醇激酶, Phosphoinositide3-kinase) 是一种胞内磷脂酰肌醇激酶, 研究证明, PI3K途径是一个 关键的信号传递转导级联, 其控制细胞生长、增殖、存活 以及细胞迁移的调节。PI3K被广泛种类的不同刺激活化, 该刺激包括生长因子、炎性介质、激素、神经递质和免疫 
球蛋白和抗原 ${ }^{[0]}$ 。PI3K有三类，I类PI3K是包含p 85 调控亚 单元和 $p 110$ 催化亚单元的异源二聚体酶, 进一步分成IA 类PI3K和IB类PI3K两类。其中, IA类PI3K的激活与受体酪 氨酸激酶密切相关。激活的PI3K催化磷脂酰肌醇第三羟基 的磷酸化, 催化在细胞膜处产生脂质第二信使磷脂酞肌醇 - 3, 4, 5-三磷酸酯 (PIP3)。PIP3可作用于多种下游效应物, 如AKT/PDK1途径中的效应物、mTOR、Tec 家族激酶和Rho 家族GTP酶等。AKT（蛋白激酶B, ProteinKinaseB）是一 种丝氨酸/苏氨酸蛋白激酶, 其N端的PH结构域可以与PI $3 \mathrm{~K}$ 产生的PI3P结合, 使AKT从细胞质转移到细胞膜上, 并在 3-磷酸肌醇依赖性蛋白激酶1（PDKI）的辅助下，通过磷 酸化AKT蛋白上的苏氨酸磷酸化位点 (Thr308) 和丝氨酸 磷酸化位点 (Ser473) , 从而激活AKT。AKT通过激活下游 的靶分子来控制细胞增殖、生存及组织血管新生 ${ }^{[10]}$ 。其中 一个重要的下游靶分子是 $\mathrm{mTOR}$ (雷帕霉素靶蛋白, mammaliantargetofrapamycin）。

\subsubsection{PI3K抑制剂和mTOR抑制剂的联用}

$\mathrm{PI} 3 K / A K T / \mathrm{mTOR}$ 途径可以认为是癌症中最常见的被 异常激活的信号通路 ${ }^{[11]}$ 。PI3K和mTOR 是通常在癌症中解 除调控的PI3K/AKT/mTOR途径中的两种主要组分。mTOR 是一种 2549-氨基酸Ser/Thr蛋白激酶，根据催化区同源 性被归类于PI3K蛋白激酶家族。mTOR以两种类型的复合 物存在, 即含有raptor亚单位的mTORC1和含有rictor的 mTORC2, mTORCl活性部分地由Akt控制, 而Akt本身部分 地由mTORC2控制。mTOR下游的效应分子包括核醣体蛋白 p70S6激酶（S6K1）和真核启动因子抑制性结合蛋白 (4E-BP1)。激活后的mT0R催化S6K1的磷酸化并激活它, 同时催化 $4 \mathrm{E}-\mathrm{BP} 1$ 的磷酸化并使其失活, 该结果导致蛋白 翻译的起始和细胞周期的前进 ${ }^{[12]}$ 。此外, mTOR还可以通 过抑制胰岛素受体底物的磷酸化和稳定性来负反馈调节 PI3K/AKT的活性。因此, 解除调控的PI3K和mTOR可协同 地用于促进肿瘤生长和存活。

目前, PI3K和mTOR抑制剂的联合给药是垂直通路联合 治疗的研究热点。文献报道, 在恶性神经胶质瘤细胞中, 雷帕霉素的TOR抑制作用与PI3K/AKT抑制作用组合可通过 扩大的自体吞噬作用而产生协同抗肿瘤作用 ${ }^{[13]}$; 通过雷帕 霉素与PI3K抑制剂LY294002组合治疗能够克服H157肺肿 瘤细胞中由Rapa诱发的eIF4E磷酸化作用, 从而增强抗肿 瘤活性 ${ }^{[14]}$; 阿里 - 扎斯克等人开发了一种具有PI3K抑制剂 活性的17-羟基渥曼青霉素衍生物, 将该衍生物之一 (1E, 4S, 4aR, 5R, 6aS, 7S) - 1 - \{[叔丁基 (2-羟乙基) 氨基] 亚 甲基 $\}-7,11$-二羟基-4-(甲氧基甲基) - $4 \mathrm{a}, 6 \mathrm{a}^{-}$二甲基 - 2, 10-二氧代-1，2，4，4a，5，6，6a，7，8，9，9a，10-十二氢荫 并 $[4,5-\mathrm{h}]$ 异苯并吡喃-5-基乙酸酯与mTOR 抑制剂CCI-779 的组合在PTEN阴性 (CCI-779敏感性) 肿瘤细胞中产生附加 的抑制作用且在结肠肿瘤细胞中产生协同抑制作用, 但所 述抑制作用与经 CCI-779或其它 17-羟基渥曼青霉素 (17-HWT) 类似物的单一药剂治疗相比具有较小敏感性 ${ }^{[15]}$; C - 加西亚一埃切夫埃里亚等人开发了一种磷脂酸肌醇 -3-激酶 (PI3K) 抑制剂 5- (2, 6-二-吗琳-4-基-嘧啶-4基)-4-三氟甲基-吡啶-2-基胺和至少一种mTOR抑制剂的 组合产品, 通过PI3K抑制剂以减少或阻断 $\mathrm{mTOR}$ 抑制剂对
AKT的磷酸化和激活, 用于治疗用 $\mathrm{mTOR}$ 抑制剂的治疗时由 于例如AKT的磷酸化和激活引起的耐药或敏感度下降的增 殖性疾病 ${ }^{[16]}$; $C$ - 弗里特什等人开发的药物组合物包括 PI3K的 a 同种型的高选择性抑制剂- - (S) - 吡咯啶 $-1,2-$ 二羧酸 2 -酰胺 1 - (4-甲基- 5 - [2- (2, 2, 2-三氟- 1,1 -二甲基 -乙基)-吡啶-4-基]-噻唑-2-基)-酰胺或其药学上可接受 的盐及至少一种哺乳动物雷帕霉素靶 (mTOR) 抑制剂或其 药学上可接受的盐, $a$ 同种型特异性磷脂酰肌醇 -3 -激酶 (PI3K) 抑制剂化合物或其药学上可接受的盐藉由mTOR抑 制剂减少或阻断AKT的磷酸化及活化。当同时、按序或分 开施用时, 该 $a$ 特异性PI3K抑制剂化合物及mTOR抑制剂相 互作用以强效抑制细胞增殖, 在治疗哺乳动物mTOR依赖性 疾病（尤其癌症）中产生出人意料的协同改良, 该有利的 相互作用可减少对各化合物所需的剂量, 导致减少副作用 及提高化合物在治疗中的长期临床效果 ${ }^{[17]}$; 任平达等人研 究了PI3K的 a 抑制剂和mTOR抑制剂的组合给药, 抑制细胞 中AKT (S473) 和AKT (T308) 两者的磷酸化, 对具有提高的 AKT磷酸化的肿瘤具有协同治疗效果 ${ }^{[18]}$ 。

\subsection{2. mTOR抑制剂与RTK抑制剂的联合给药}

PI3K/AKT/mTOR信号通路被RTK（受体酪氨酸激酶 (receptortyrosinekinase)) 激活。受体酪氨酸激酶包 括胰岛素受体 (IR), 胰岛素样生长因子受体 (IGF-1R), 血小板衍生生长因子受体 (PDGFR) 以及表皮生长因子受 体（EGFR）。受体酪氨酸激酶可以直接地调节PI3Kp 85 亚 基或者间接通过胰岛素受体底物（IRS）调节与其相互作 用的PI3Kp85亚基来激活PI3K ${ }^{[19]}$ 。

\section{(1) IGF-1R抑制剂}

PI3K信号传递途径接受来自配体-受体相互作用（诸 如EGFR和IGF-1R）的上游输入, IR和IGF-1R的信号引起胰 岛素受体底物（IRS）磷酸化, 磷酸化后的胰岛素受体底 物转而与p 85 亚基的SH2结构相互作用, 导致招募PI3K到细 胞膜, 并且释放p85亚基对PI3K抑制, 从而激活PI3K, 并 通过诸如雷帕霉素的哺乳动物靶标 (mTOR) 等下游效应物 发信号。已证实mTOR抑制可以诱导能够引起癌细胞中AKT 激活的上游IGF-1R信号传导。通过抑制PI3K轴中的上游和 下游分子靶标, mTOR和IGF-1R抑制剂的组合可以提供协同 效应。S . 萨思亚纳拉亚南等人使用 $\mathrm{mTOR}$ 抑制剂和抗 -IGF-1R抗体治疗选自下述癌症的方法: 非小细胞肺癌、乳 腺癌、结直肠癌、软组织或骨肉瘤和子宫内膜癌, 其中所 述mTOR抑制剂选自:ridaforolimus、依维莫司、坦罗莫司 和它们的组合, 且所述抗-IGF-1R抗体选自: dalotuzumab、 figitumumab、cixutumumab、SHC717454、RocheR1507和 AmgenAMG479 ${ }^{[20]}$ 。

\section{（2） EGFR（例如Her-2、EGFR）}

EGFR是PI3K-AKT-mTOR通路激活的膜受体, 在许多肿 瘤中有较高频率的突变, 由其引发的通路过度激活与肿瘤 病理和预后有着密切的关系。阿里 - 扎斯克等人公开了具 有 PI3K 抑制剂活性的渥曼青霉素衍生物 (1E, $4 \mathrm{~S}, 4 \mathrm{aR}, 5 \mathrm{R}, 6 \mathrm{aS}, 7 \mathrm{~S})-1$ - $\{$ [叔丁基 (2-羟乙基) 氨基] 亚 甲基 $\}-7,11$-二羟基 -4 - (甲氧基甲基)- $4 \mathrm{a}, 6 \mathrm{a}-$ 二甲基 
- 2,10 -二氧代- $1,2,4,4 a, 5,6,6 a, 7,8,9,9 a, 10$-十二氢狮 并 $[4,5-\mathrm{h}]$ 异苯并吡喃-5-基乙酸酯与 HER $2 /$ neu 抑制剂 HKI-272 的组合对过度表达HER2/neu的MDA361-DYT2乳房 肿瘤细胞中的协同抑制作用 ${ }^{[15]}$ 。

\section{2. 3. $P D K 1$ 抑制剂和 $m T O R$ 抑制剂的联合给药}

于强等人研究了PDK1表达和/或活性的抑制剂和mTOR 抑制剂的组合在制备用于治疗或预防癌症的药物中的应 用 ${ }^{[21]}$ 。

\section{3. JAK-STAT通路}

JAK-STAT系统是第二信使系统的主要替代信号转导 途径, 由受体、JAK (Janus酪氨酸激酶) 和STAT (信号转 导及转录活化因子) 蛋白三个主要成分组成。所述受体由 来自干扰素、白介素、生长因子、或其它化学信使的信号 激活。JAK家族目前已确定包括JAK1、JAK2、JAK3和TYK2 激酶, 形成一个胞内蛋白酪氨酸激酶家族; 这些激酶对调 节细胞因子受体信号转导发挥着重要作用, 所述信号转导 诱导包括细胞增殖、分化和细胞存活在内的多种生物反应。 STAT家族共有STAT1、STAT2、STAT3、STAT4、STAT5a、STAT5b、 STAT6。JAK-STAT信号通路经细胞膜将信息从细胞外的化 学信号递送到细胞核中DNA上的基因启动子, 引起该细胞 中的DNA转录和活性。受体与配体的结合可激活JAK的激酶 功能, 随着激酶活性增加, 其使受体上的酪氨酸残基磷酸 化并产生与蛋白相互作用的位点, 这些蛋白包含结合磷酸 酪氨酸的SH2结构域。能结合这些磷酸酪氨酸残基的具有 SH2结构域的STAT被募集到所述受体, 并通过JAK自身酪氨 酸磷酸化。然后, 这些磷酸酪氨酸用作其它STAT的SH2结 构域的结合位点, 调节其二聚化。不同的STAT形成杂或同 二聚体。活化的STAT二聚体在细胞核中积聚并激活其靶基 因转录。STAT还可直接通过受体酪氨酸激酶如表皮生长因 子受体以及通过非受体酪氨酸激酶如 $c-S r c$ 来进行酪氨酸 磷酸化。波士顿生物医药公司的专利中, 应用了Stat3通 路抑制剂和包括选自表皮生长因子受体 (EGFR) 或血管内 皮生长因子受体 (VEGFR) 的生长因子受体靶向剂、酪氨酸 激酶抑制剂的第二药剂组合治疗受试者癌症 ${ }^{[22]}$ 。

\section{4. Ras/Raf/MEK/ERK通路}

有丝分裂原活化蛋白激酶（mitogen-activated protein kinase, MAPK) 信号级联放大转导肿瘤细胞内的 多种增殖和分化信号。例如, 该途径负责转导表皮生长因 子 (EGF) 的信号从而促进细胞增殖, 将EGF的最初胞外信号 转换成对细胞周期进程至关重要的基因转录改变。其中的 $\mathrm{Ras} / \mathrm{Raf} / \mathrm{MEK} / \mathrm{ERK}$ 信号级联通路可被广泛激活, 由一个小 GTP结合蛋白连接活化的受体酪氨酸激酶和胞浆蛋白激酶 级联反应。其活化的中心是使Ras进行鸟苷酸交换变成其 活化形式RasGTP。该反应通过鸟苷酸交换因子SOS与回到 酪氨酸磷酸化受体上的结合蛋白组成复合物或形成 SHC-Grb2-SOS复合物而进行。Ras蛋白几乎可被所有细胞 因子受体激活, 当Ras变异时, 可引起细胞的异常增殖, 导 致肿瘤的发生。胞外信号调控激酶 (ERK) 是Ras下游效应子, 在Ras和ERK之间还存在其他相关的效应子, 相互之间构成
了一条完整的Ras/Raf/MEK/ERK信号通路。联合给药通过 交叉影响Ras/Raf/MEK/ERK信号通路与其他细胞内信号通 路发挥协同作用。

葛兰素史克公司、赛诺菲公司等多家跨国制药公司均 针对在癌症治疗中组合使用PI $3 K-A K T$ 通路调节剂和 $\mathrm{RAF} / \mathrm{MEK} / \mathrm{ERK}$ 通路调节剂提出了专利申请 ${ }^{[23]-[28]}$, 所述组合 用药包括了PIK3抑制剂(如索拉非尼、Raf265、SB590885、

XL281、PLX4032、GDC-0941、（S) -1-(4-( (2- (2-氨基嘧啶 -5-基)-7-甲基-4-吗啉代噻吩并 [3, 2-d] 嘧啶-6-基) 甲基） 哌嗪 -1 - 基 ) -2 - 羟基丙 -1 - 酮 ）和 MEK 抑制剂（如 GDC-0973/XL-518 、PD325901 、 PD-181461 ARRY142886/AZD6244、ARRY-509、XL518、JTP-74057、 AS-701255、AS-701173、AZD8330、ARRY162、ARRY300、 RDEA436 、E6201 、R04987655/R-7167、GSK1120212 、 AS703026) 的组合, MEK抑制剂 $N-\{3-[3$-环丙基 $-5-(2-$ 氟 -4-碘-苯基氨基) - 6 , 8-二甲基- 2 , 4, 7-三氧代- 3 , 4, 6, 7四氢 $-2 \mathrm{H}$-吡啶并 $[4,3-\mathrm{d}]$ 嘧啶-1-基]苯基 $\}$ 乙酰胺和 $A k t$ 抑 制剂 $\mathrm{N}-\{(1 \mathrm{~S})-2$-氨基 $-1-[$ ( 3,4 -二氟苯基) 甲基 $]$ 乙基 $\}-5-$ 氯-4-(4-氯-1-甲基- $1 \mathrm{H}$-吡唑-5-基) - 2 -呋喃甲酰胺的组 合等。此外, 葛兰素史克公司还对MEK抑制剂的其他组合 给药, 如mTOR抑制剂提出了申请 ${ }^{[29]}$ 。结果显示, 组合用药 发挥了协同作用, 与单独使用任一化合物进行治疗的肿瘤 相比, 肿瘤生长减少了更多。

\section{4. 结语}

靶向药物的联合用药虽然将是未来抗肿瘤开发的一 个亮点，但在技术上、商业上亦有多方面的挑战:

首先，篎选联合用药的协同效应、相加甚至拮抗作 用不仅代表肿瘤生物学的水平, 很多情况下甚至有很多 偶然性, 还可能会因为药物之间的相互作用或者抗癌药 物作用的周期特异性对疗效和毒性产生影响, 如何预测 或评价药物间的相互作用, 选择合适的配伍药物、设计 用药顺序, 调节药物组分的比例等都是必须克服的问题。 肿瘤的发生和发展是一系列的分子事件: 信号转导异常、 表皮生长因子受体失调、血管生长因子的异常分泌、癌 基因的激活和抑癌基因失活和微小RNA的异常表达等, 与 这些分子相关的因素和信号通路间的相互作用构成复杂 的网络系统。应选择什么样的靶点和 (或) 通路, 应靶向 作用于垂直或平行通路, 通路之间的相互联系和串扰都 是药物开发需要考虑的问题。此外, 不同致病因素和有 害习惯可导致不同基因的突变或失活, 可触发不同信号 通路促进恶性肿瘤的发生和发展, 这些患者个体化的差 异也增加了联合用药的复杂性。

其次, 相对来讲联合用药的开发成本更高, 成功的不 确定性较大。因为联合用药的临床实验至少需要单药给药、 联合用药等三个或更多的单臂治疗组, 病例数因此也远远 高于单药实验, 开发成本相应地也显著增加。尽管在临床 实验前已经做过很多体外和临床前动物实验的验证工作, 但是这些实验是否能转化到临床还有很大的不确定性, 两 种药物的协同效应才有望明显提高疗效而不明显增加其 毒副作用。 
最后，专利保护是对投资巨大医药开发的首要保障， 不同公司之间的开发协议一直是开发靶向药物联合给药 的主要障碍, 联合试验可能会产生新的知识产权问题。联 合用药的保护范围有较大的局限性, 不仅包括用途的可专 利性, 也牵扯到潜在专利侵权和诉讼。由于抗癌药物的技 术准入门槛较高, 重点药物主要集中在少数几个拥有专利 的国外大型企业手中, 导致行业集中度较高, 呈现出高度 寡占型的行业特征。然而随着近年重磅药物的专利到期, 以及来自仿制药的冲击, 使得抗癌药物的行业集中度有所 下降。由于国外抗肿瘤多靶向治疗的研发速度很快, 虽然 中国对于联合给药的研发也加快了速度, 但是在专利领域 的整体研究和分析仍属空白, 很大程度上制约了中国在该 领域的技术发展速度。因此, 有必要及时在该领域开展专 利分析和研究, 把握国际前沿技术和发展趋势, 研究克服 知识产权和监管壁垒, 为中国企业在该领域的创新性研发 和市场运作提供技术指导以及可行的专利发展战略, 帮助 中国企业在激烈的国际竞争中谋求发展空间。

尽管实现联合靶向治疗的过程中还存在许多挑战和 困难，仍可以预计在未来的肿瘤药物发展中，除了具有特 异性与靶向性的抗癌药物会继续发展外, 癌症靶向药物之 间的联合治疗也将成为抗癌药物研究的主要方向。

\section{参考文献}

１1］世卫组织预计到2030年癌症死亡人数可能翻番, 生物学通 报， 2010年第45卷第03期， 25 。

[2] 胡胜等, 癌症靶向药物的联合治疗, 药品评价, 2012年第9 卷第21期, 28-35。

[3] Mendelsohn J, et al., Status of epidermal growth factor receptor antagonists in the biology and treatment of cancer. J. Clin. Oncol., 2003, 21(14): 2787-2799.

４］］王预等, 厄洛替尼与西妥昔单抗联合应用治疗吉非替尼耐 药N S C L C 的疗效, 中国肿瘤临床, 2011年第38卷第24 期， 1505-1509。

[5] 韦玮等, 西妥昔单抗联合厄洛替尼对人肝癌细胞的体外抑 制作用, 癌症, 2008年第4期, 386-392。

[6] Miller KD, et al., Randomized phase III trial of capecitabine compared with bevacizumab plus capecitabine in patients with previously treated metastatic breast cancer, J. Clin. Oncol., 2005, $23(4)$ : 792-799.

[7] Blackwel1 KL, et al., Randomized study of Lapatinib alone or in combination with trastuzumab in women with ErbB2-positive, trastuzumab-refractory metastatic breast cancer, J. Clin. Oncol., 2010, 28(7) : 1124-1130.

８］江秉华 等，CN101332301A，2008年12月31日。
[9] Wymann, M. P., et al., Phosphoinositide 3-kinase signalling - which way to target Trends Pharmacol. Sci., 2003, 24(7): 366-376.

[10] Cantley, C. C., et al., New insights into tumor suppression: PTEN suppresses tumorformation by restraining the phosphoinositide 3-kinaseyAKT pathway, Proc. Natl. Acad. Sci., 1999, 96: 4240-4245.

[11] Engelman, J. A., Targeting PI3K signalling in cancer:opportunities, challenges andlimitations, Nature Reviews/Cancer, 2009, 9: 550-562.

[12] Kozma, S. C. et al., Regulation of cell size in growth, development and human disease:PI3K, PKB and S6K, Bioessays, 2002, 24: 65-71.

[13] Takeuchi H., et al., Synergistic Augmentation of Rapamycin-Induced Autophagy in Malignant Glioma Cells by Phosphatidylinositol3-Kinase/Protein Kinase B Inhibitors, Cancer Res., 2005, 65 (8) : 3336-3346.

[14] Sun S. Y., et al., Activation of Akt and eIF4E Survival Pathways byRapamycin-Mediated Mammalian Target of RapamycinInhibition, Cancer Res., 2005, 65(16): 7052-7058.

[15] BURSAVICH M. , et al. , W02006044453A1，2006年4月 27 日。

[16] GARCIA E. C. , et al. , W02010049481A1，2010年5月6日。

[17] FRITSCH C. , et al. , W02012148846 A1，2012年11月1日。

[18] GU0 X., et al. , W02012148540 A1，2012年11月1日。

[19] B. Markman, et al., Status of PI3K inhibition and biomarker development in cancer therapeutics, Ann. Oncol., 2009, 21(4): 683-691.

[20] KLINGHOFFER R., et al. , W02010120599 A2 2010年10月 21日。

[21] TAN J., et al., W02011084108 A1，2011年7月14日。

[22] LEE C. J., et al. , W02009036101A1，2009年3月19日。

[23] AFTAB D., et al., W02008021389 A2，2008年2月21日。

[24] FRITSCH C. , et al. , W02010006225 A1，2010年1月14日。

[25] DUMBLE M., et a1., W02011038082 A1，2011年3月31日。

[26] DUMBLE M. , et al., W02011038085 A1，2011年3月31日。

[27] BEL V. M. , et al., W02011054620 A1，2011年5月12日。

[28] BIHONGNEU 0. A., et al., W02013037943 A1，2013年3 月21日。

[29] GILMER T., et a1. , W02011062930A1，2011年5月26日。 